\title{
A performance investigation on IoT enabled intra-vehicular wireless sensor networks
}

\section{Md. Arafatur Rahman, Jahan Ali, Muhammad Nomani Kabir*and Saiful Azad}

\author{
University Malaysia Pahang \\ Faculty of Computer Systems and Software Engineering \\ Gambang, 26300, Malaysia. \\ *Email: arafatur@ump.edu.my,nomanikabir@ump.edu.my \\ Phone +609-5492231
}

\begin{abstract}
The concept of Internet of Things (IoT) can be utilised in vehicles, since the number of sensor nodes in vehicles is rising tremendously because of the uplifting demand of applications for security, safety and convenience. In order to establish the communication among these nodes inside a vehicle, a controller area network with wired architecture provides a prominent solution. However, this solution is not flexible because of the architectural complexity and the demand for a large number of sensors inside the vehicle; hence wired architectures are replaced by wireless ones. Moreover, scalability will be an important issue while introducing the IoT concept in Intra-Vehicular Wireless Sensor Networks (IVWSNs). In this paper, a comprehensive performance investigation on the IoT enabled IVWSNs (IoT-IVWSNs) to be carried out in order to address this issue. The overview of the IoT-IVWSNs with a comparative study of the existing technologies and the design challenges for such network are provided. The link design between an enddevice and the control unit is analysed, and the performance of the network has been investigated and some open research issues are addressed. It reveals that the delay in packet transmission increases due to higher traffic loads and the number of end-devices. This result demonstrates that the existing MAC protocol works well for a small network (i.e., a network with a maximum number of 50 nodes) but is not suitable for a large network (i.e., a network with more than 50 nodes). The outcome of this research helps to design a smart car system.
\end{abstract}

Keywords: IoT; Intra-vehicular wireless sensor network; scalability; congestion.

\section{INTRODUCTION}

The Internet of Things (IoT) is an environment where objects are tagged with smart devices (e.g. sensor, actuator, etc.) having unique identifiers and being able to transfer information throughout the network without involving human interaction [1-3]. It is an emerging concept that introduces several new paradigms in communication such as the Device-to -Device (D2D) [4], or Machine-to-Machine (M2M) [5] communications. It can also be enabled by existing networks (e.g. Monitoring and Rescuing Network, Intravehicular Network, Inter-vehicular network, etc.) in order to design fully automated smart systems. The integration of the IoT and intra-vehicular network will be focused in this paper. 
The Controller Area Network (CAN) is one of the widely used communication protocols, especially for wired intra-vehicular communications [6]. The serial communication paradigm makes it highly secured and efficient for real-time systems. However, the CAN is not flexible for high network nodes (devices) because of the architectural complexity inside the vehicles; hence wired architectures are replaced by wireless ones [7-9]. The rapid increase of sophisticated electronic systems inside modern vehicles increases the necessity of deploying a higher number of sensors to monitor them. To fulfil this aim, the concept of IoT can be introduced in intra-vehicular communication, referred as IoT enabled Intra-Vehicle Wireless Sensor Networks (IoT-IVWSNs). It is a network where a large number of sensors are connected to each other in order to share the car-status so as to develop a smart car system. Moreover, scalability will be an important issue while introducing the IoT on IVWSNs. Different networking devices e.g., UWB, RFID, Wi-Fi, Bluetooth and ZigBee can be deployed inside a vehicle to facilitate the communication. A large number of sensor nodes are placed to monitor the vehicle safety components (e.g. engine oil, engine temperature, tire pressure, fuel level, car interior temperature, door locks, speed). These sensor nodes can transmit and receive the information of the safety status of the components in the IVWSN. While introducing the IoT concept with IVWSNs, it will be an important factor to investigate whether the existing MAC protocol can provide a suitable solution for such IoT enabled IVWSNs. It is expected that the existing protocol will not perform better due to the collision and congestion of the network because of the high traffic loads that occur due to communication among many devices. In order to mitigate these issues, a new CSMA/CA MAC protocol strategy needs to be designed. The problem will be considered while designing the network scenario and providing optimum solutions to design an intelligent vehicle system.

A lot of works have been conducted on vehicular communications specifically for inter-vehicular, intra-vehicular and vehicle to infrastructures wireless-sensor-networks (WSNs). Due to the uplifting demand of driver safety and assistance in a modern vehicle, the intra-vehicle WSNs have become a more interesting research area nowadays. In [10], the authors investigated the performance of intra-vehicle communication in terms of the different number of time bandwidth and transmitted pulses per symbol condition exploiting the UWB technology. In [11], the authors evaluated the channel capacity of the UWB with a multi-antenna approach for a high speed intra-vehicle wireless communication system. They also compared different UWB pulse modulation schemes for finding a suited modulation scheme for in-vehicle communication systems in [12]. Again in $[13,14]$, the authors discussed the small and large scale path loss statistics using the UWB band deployed sensors in different locations inside a car. In [15], the authors used the UWB channel in IVWSNs for investigating the optimal power control, rate adaptation and scheduling in one Electronic Control Unit (ECU) and multiple ECU cases.

Several works have been done exploiting the RFID technology for Intra-vehicular communication. In [16], the authors proposed an in-vehicle sensors fusion strategy for positioning the vehicle that is completely under a GPS defined environment using the RFID technology protocol. In [17], the authors investigated the energy harvesting protocol for wireless sensor networks and they proposed the RFID passive tag for renewable and sustainable energy harvesting for wireless sensor networks. In [18], they investigated how to design an efficient protocol for collecting data from the deployed sensors in numerous locations in large scale RFID systems. In [19], the author proposed an algorithm for a traffic monitoring system using combined active RFID and GSM technology. Moreover, the Bluetooth technology has also been introduced for such 
communication systems. In [20], the authors proposed a solution to reduce the power consumption of the sensor nodes using Bluetooth technology for in-vehicle wireless communication. In [21, 22], the authors reported that Bluetooth consumes low energy levels for the intra-vehicle wireless communication system. However, due to the poor efficiency and reduced reliability of the MAC protocol, requiring a high power level, supporting a number of insufficient end-nodes, and propagation and security problems, the above technologies are not suitable for intra-vehicle wireless sensor networks especially for comparatively large networks [23-25].

On the other hand, the ZigBee communication protocol is a viable and prominent technology for intra-vehicular communication that provides a real-time monitoring system to design low cost, low data rate and low power wireless communication systems. In [24], the authors investigated the radio channel of ZigBee in a complex vehicular environment. In [26], the authors examined the versatile platform for IVWSNs which enables side blind zone alert systems for other vehicles. Other authors proposed remote monitoring and controlling systems for home networks based on the ZigBee technology with the help of web services in [27]. In [28], the authors showed that the ZigBee communication protocol is the reliable transmission protocol for patient monitoring systems. In [29], the author analysed the link and network reliability for a ZigBee based intra-vehicle wireless sensor network. In [30], they use ZigBee for tracking the vehicular positional coordinate with the aid of Vehicular Ad-hoc Networks (VANETs). ZigBee is a viable and robust type of technology to design intra-vehicle wireless sensor networks, as analysed by [31, 32]. All the aforementioned works are applicable for small networks, where the numbers of end-nodes are limited. The IoT concept needs to be incorporated with intra-vehicular communication, since various types of sensor nodes are increasing day by day inside the vehicle for the demand of driver assistance, fatigue detection, road safety, and so on. Recently, researchers estimate that the numbers of sensor nodes inside the vehicle will be significantly increased by 2020 [24, 25]. Establishing reliable communication for such scalable network will be challenging due to the internal complexity inside the vehicle. As a consequence, it is an important issue to investigate the performance of such network by exploiting the ZigBee technology. Hence, in this paper, we have conducted an extensive investigation for IoT enabled intra-vehicular networks and addressed several issues and challenges. In this paper, a comprehensive performance investigation on IoT enabled IVWSNs (IoT-IVWSNs) has been carried out in order to address this issue. First, we discuss the overview of IoT-IVWSNs. Next, a comparative study of the existing technologies and the design challenges for such network is provided. Then, the link design between the end device and the control unit is analysed. Furthermore, the network performance is investigated. Based on the investigation, some open research issues are addressed. The rest of the paper is organised as follows. In Section 2, we discuss the related works. We describe the overview of IoT-IVWSNs in Section 3. In Section 4, we investigate the network performance and address some open research issues. Finally, Section 5 concludes the paper.

\section{METHODS AND MATERIALS}

We concentrate on the main component of the IoT-IVWSNs. Indeed, many car manufacturers install wireless connectivity technologies in their vehicles to facilitate communication between vehicles and also with the infrastructure. Intra-vehicular Wireless Sensor Networks (IVWSNs) are designed on top of these vehicular networks by equipping vehicles with onboard sensing devices. In such case, the sensors can gather a 
set of information such as video data, speed, localisation, acceleration, temperature, seat occupation, etc. The basic idea of IoT-IVWSNs is to deploy traffic sensor network applications so as to monitor the critical parts of the vehicle in order to create a fully automatic vehicle system for the vehicle in the near future. An overview of the IoTIVWSNs is presented in this section. We begin with the introduction of the IoT concept on IVWSNs. Then, we discuss the architecture and the components of this network and finally, the suitable existing technologies and the challenges for this network are discussed.

\section{IoT on IVWSNs}

The IoT can accommodate various nodes to be sensed and controlled remotely within the existing communication infrastructure. It makes an integration of a computer based system directly with the physical world. As a result, the efficiency, accuracy, the complexity of maintenance, and economic benefit of the system improve significantly. Moreover, nowadays, modern vehicles are equipped with various types of sensors for improving driver safety, convenient transportation and traffic monitoring systems. Consequently, the numbers of sensor nodes inside vehicle are increasing day by day. The existing wired Electronic Control Unit (ECU) device and subsystem integrated with Controller Area Network (CAN) has some problems due to the limiting range of sensor installation, and the network complexity. With the increase of sensors inside a vehicle, more wire connections are required for implementation. As a result, the weight, complexity and manufacturing cost significantly increase. Due to these reasons, the IntraVehicle Wireless Sensor Networks (IVWSNs) have recently been introduced to minimise the above complexity in the automotive industries [10,13]. With the help of wireless technologies inside a vehicle, the weight, cost and fuel consumption of the vehicle can be reduced and a better performance can be achieved. Therefore, a large number of nodes need to be integrated with each other for reliable and efficient communications. To realise this aim, in this paper, the IoT concept is taken into account for designing IVWSNs referred to as IoT enabled IVWSNs (IoT-IVWSNs), as shown in Figure 1. IoT-IVWSNs can be defined in Definition 1. Definition 1 (IoT-IVWSNs): The term refers to the network where a large number of sensors retrieve the status of the critical components of the vehicle and transmit them to the central entity in order to monitor the functionality of the system.

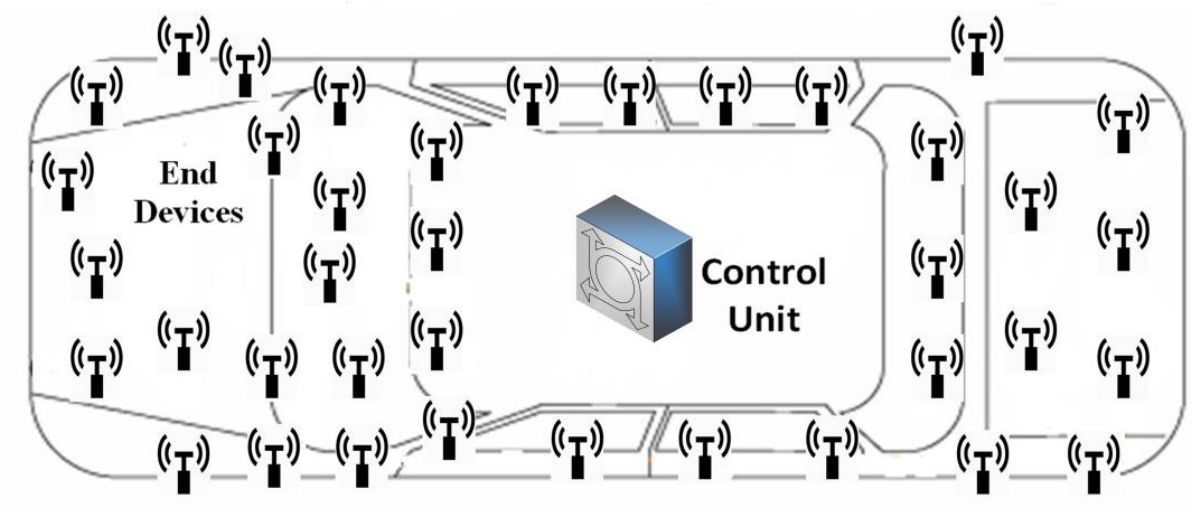

Figure 1. IoT enabled IVWSNs scenario.

In the following, we briefly discuss about the network components, network architecture and data flow of the network. 


\section{Network Components}

We will discuss the main and the sub-components of the network. The network consists of mainly three components, namely the end-device (ED), control unit (CU) and display. Each ED is embedded with a transmitter and a specific sensor. Different types of sensors are installed (e. g. temperature sensor, speed sensor, defect sensor, water sensor, heat sensor, tire-pressure monitoring sensor, parking sensor, knock sensor, etc.) inside a vehicle to collect the current status of the critical components of a vehicle [33-36]. The sensors sense the information of a component and the transmitter, which is integrated with the sensor, transmits the information to the CU. The CU also contains two types of subcomponents such as the Transceiver and Processing Unit (PU). The Transceiver can receive and send data. The PU can make the decision whether the data will be sent to the display or not. If the new information is different from the old one, then it will send the result to the display through the Transceiver of the CU. The display will show the current status of the different components of the vehicle.

\section{Network Architecture}

The performance and reliability of a network depend on the proper design and the architecture of the network. Since the complexity of the network is increasing, future intra-vehicular communication needs to be properly designed. There are critical and hostile environments inside the vehicle [37-39]. So the network architecture should be adjusted. In our network architecture diagram (as depicted in Figure 2(a)), we can see that there are two tiers of network, one is the information acquisition and another one is the information processing. The information acquisition section refers to collecting data from various components of the deployed sensors inside the vehicle. Then, the acquired data is received by the $\mathrm{CU}$ and processed by the PU in order to know the status of the car. Here, we consider the single hop star topology for the network. As a result, each and every ED can communicate with the same signal strength. The main advantage of this topology is to reconfigure the network to skip broken nodes and it is possible to choose the shortest path to a certain destination. Moreover, the data flow of the network is shown in Figure 2(b).

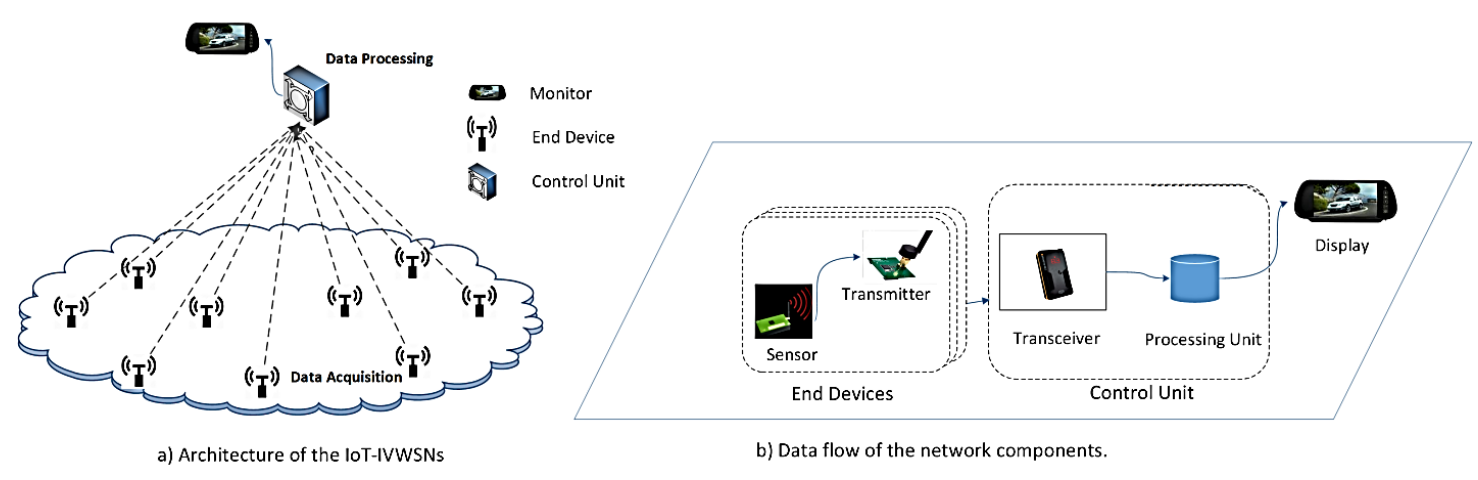

Figure 2. (a)Network architecture; and (b) data flow.

\section{Existing Technology for IoT-IVWSNs}

Many wireless sensor technologies have been introduced in recent years, such as Bluetooth, Wi-Fi, UWB, ZigBee and RFID. Each and every technology has specific criteria to operate in a suitable area. Specifically, Bluetooth is a short range wireless type of technology complying with the IEEE 802.15.1 standard. It can communicate up to 
$3 \mathrm{Mb} / \mathrm{s}$ in portable devices. The transmission needs a high power level so that it is not appropriate for battery-enabled sensors in vehicles. Again, the Ultra-Wideband (UWB) is a radio technology, which operates with a 3.1-10.6 frequency band and supports a short range communication data rate up to $480 \mathrm{Mb} / \mathrm{s}$. The RFID PHY layer waveform and simple MAC protocol design (coupled with high cost of RFID readers) make it unsuitable for WSN reliability and latency requirements [40]. Again, ZigBee is a short range communication form of technology maintaining the IEEE 802.15.4 standard [41, 42]. It operates on the ISM radio spectrum $(868 \mathrm{MHz}, 915 \mathrm{MHz}$, and $2.4 \mathrm{GHz})$. It is characterised by low cost, high data rate, low power consumption, and supports the star, mesh and hybrid topology. It has a long battery life and provides more security by its encryption technique. The ZigBee transmission power is $-25 \mathrm{dBm}$, which is significantly lower than others. Moreover, it needs only $15 \mathrm{~ms}$ to wake up. This emerging feature allows the transmission device to remain most of the time in sleeping mode, which preserves the device's battery power. In [43], they have evaluated the performance for the intra-car wireless sensor network. This study showed that the ZigBee is a viable and promising technology for intra-car wireless sensor networks. ZigBee is designed to last for six months to two years on just two AA batteries [25]. Hence, the IEEE 802.15.4 protocol is a good candidate to design an IoT application with low power consumption. As a result, the ZigBee is expected to play an important role in the design of IoT-IVWSNs for future generations. Moreover, the ZigBee supports different types of network topologies and its MAC protocol is designed with CSMA/CA features that provide extra facilities to ensure data transfer robustly. The main advantage of this protocol is to allow large sensor nodes in a single network. The number of sensor nodes inside a vehicle continues to increase every year. So, this huge number of sensor nodes can be maintained through this protocol. Table 1 summarises the main differences of existing wireless protocols that are the IEEE standard.

Table 1. Comparison of existing technologies for IoT-IVWSNs.

\begin{tabular}{|c|c|c|c|c|}
\hline Standard & Bluetooth & $\mathrm{Wi}-\mathrm{Fi}$ & UWB & ZigBee \\
\hline IEEE specification & 802.15 .1 & $802.11 \mathrm{a} / \mathrm{b} / \mathrm{g}$ & $802.15 .3 a$ & 802.15 .4 \\
\hline Frequency band & $2.4 \mathrm{GHz}$ & $\begin{array}{c}2.4 \mathrm{GHz} ; 5 \\
\mathrm{GHz}\end{array}$ & $\begin{array}{c}3.1-10.6 \\
\mathrm{GHz}\end{array}$ & $\begin{array}{c}\text { 868/915 GHz; } 2.4 \\
\mathrm{GHz}\end{array}$ \\
\hline Max signal rate & $1 \mathrm{Mb} / \mathrm{s}$ & $54 \mathrm{Mb} / \mathrm{s}$ & $110 \mathrm{Mb} / \mathrm{s}$ & $250 \mathrm{~Kb} / \mathrm{s}$ \\
\hline Nominal range & $10 \mathrm{~m}$ & $100 \mathrm{~m}$ & $10 \mathrm{~m}$ & $10-100 \mathrm{~m}$ \\
\hline $\begin{array}{l}\text { Max number of cell } \\
\text { nodes }\end{array}$ & 8 & 2007 & 8 & $>65000$ \\
\hline Chip price & $\$ 5$ & $\$ 20-25$ & $\$ 1$ & $\$ 2$ \\
\hline Data Protection & $\begin{array}{l}\text { 16-bit } \\
\text { CRC }\end{array}$ & 32-bit CRC & $\begin{array}{l}\text { 32-bit } \\
\text { CRC }\end{array}$ & 16-bit CRC \\
\hline Topology & Star & ESS,BSS & $\begin{array}{l}\text { Peer-to- } \\
\text { peer }\end{array}$ & $\begin{array}{l}\text { Tree, star, mesh, } \\
\text { and hybrid. }\end{array}$ \\
\hline
\end{tabular}

Challenges for IoT-IVWSNs

The design challenges for IoT enabled IVWSNs are discussed as follows: 
- A large number of sensors need to be considered compared to the traditional IVWSNs; therefore, the design of a scalable network for IVWSNs will be a big challenge.

- Due to the small-scale fading, three different propagation mechanisms (such as reflection, diffraction and scattering) can change the behaviour of the transmitted signal from the transmitter to the receiver inside the vehicle. Hence, these mechanisms have to be considered while designing an IoT-IVWSN.

- The wireless channel can be characterised by two fading distribution functions, such as Rice (it occurs in presence of Line of Sight (LOS) propagation path) and Rayleigh (it occurs in presence of Non LOS (NLOS) propagation path) distributions. However, these traditional concepts may not work properly, because of the architectural complexity inside the vehicle. Therefore, the actual distribution needs to be estimated through the experimental analysis that introduces an additional design challenge for IoT-IVWSNs.

- In order to design the link between the transmitter (Tx) and the receiver (Rx), the communication parameters (transmit power, received signal threshold, distance between $\mathrm{Tx}$ and $\mathrm{Rx}$, the value for the path-loss exponential, etc.) need to be set properly.

- Selecting the suitable technology for designing an IoT-IVWSN from existing technologies, requires a comprehensive analysis. In addition, new technology could be thought for such a network.

\section{RESULTS AND DISCUSSION}

In this section, first, we design the link between the ED and CU in order to set the communication parameters, and then we investigate the performance of IoT-IVWSNs considering network scalability.

\section{Link analysis between the End-Device and the Central Entity}

In this sub-section, we analyse the link between the ED and CU of the IoT-IVWSNs in terms of throughput and received power. To this end, we have examined through a discrete event network simulator named OPNET. The ZigBee network model is integrated with OPNET. First we define the basic terminologies: pass loss and throughput.

At distance d, path loss $P_{L}(d)$ is defined as

$$
P_{L}(d)=P_{t} / P_{r}>1
$$

where $P_{t}=$ Transmitted power and $P_{r}=$ Received power.

The above equation can be expressed in $\mathrm{dB}$ units as

$$
P_{L}(d)[d B]=10 \log _{10}(P L / P r)>0
$$

and

$$
P_{L}(d)[d B]=P_{L}\left(d_{0}\right)[d B]+10 \gamma \log _{10}\left(d / d_{0}\right)+X \sigma
$$


where $X \sigma$ is a Gaussian random variable, $\mathrm{N}\left(\mathrm{O}, \sigma^{2}\right)$, with zero mean and variance $\sigma^{2}$, also known as lognormal shadowing, $\mathrm{PL}\left(\mathrm{d}_{\mathrm{o}}\right)$ is the path loss in $\mathrm{dB}$ at the reference distance $\mathrm{d}_{\mathrm{o}}$ and $\gamma$ is the path loss exponent.

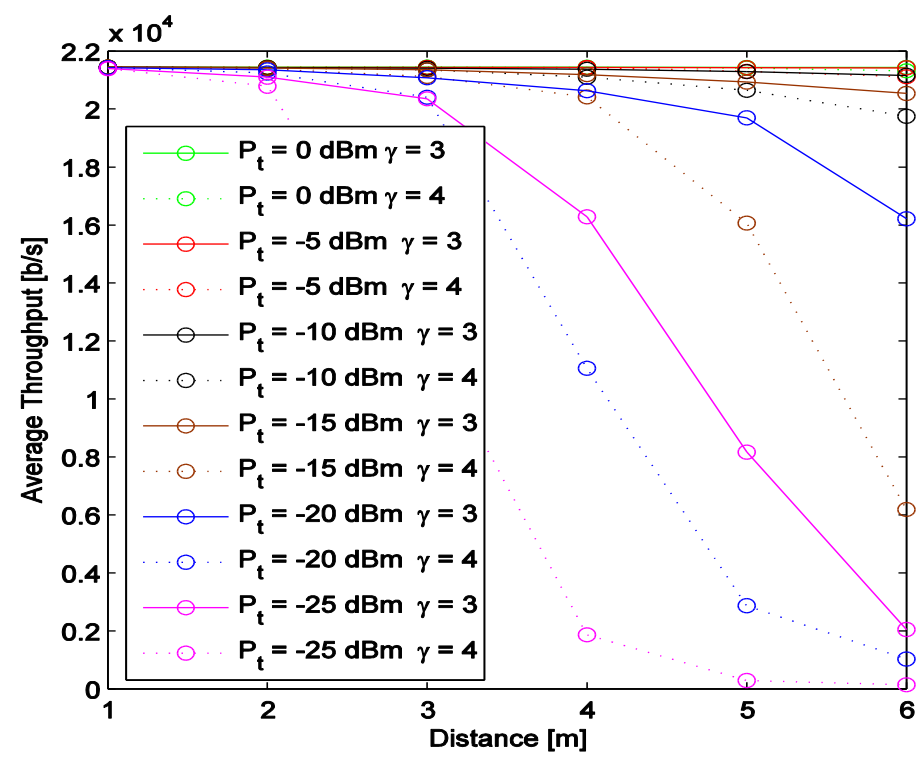

(a)

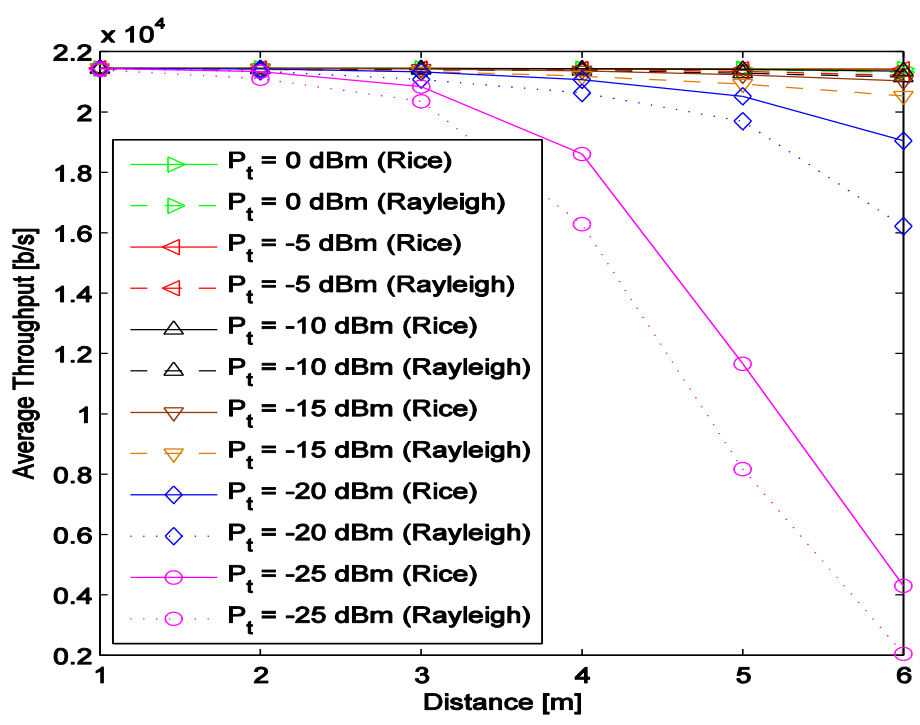

(b)

Figure 3. Performance investigation of IoT-IVWSNs in terms of throughput (a) Average throughput Vs d: varying the Tx power; (b) Average throughput Vs d: varying the Tx power and presence of the Rice and Rayleigh fading distribution function.

The performance of this model not only depends on the distance between the transmitter and the receiver, but also on the path loss exponent and the variance of the lognormal shadowing. Throughput is defined as the total number of data traffic successfully delivered to the 802.15.4 MAC layer of the receiver and sent to the higher levels in per unit time. The ED and CU are placed at a distance d, where Distance Set: $\{1$, 
$2,3,4,5,6\}$. We also consider a set of Transmit Power (denoted by Pt) (i.e., $\{0,-5,-10$, $-15,-20,-25\} \mathrm{dBm})$, as it is suited for ZigBee [23]. The considered Carrier Frequency (Cf) is $2.4 \mathrm{GHz}$. We also consider both Rice and Rayleigh fading distribution functions in order to analyse the LOS and NOLOS propagation paths where the value of $\mathrm{K}$ is 20.16 $\mathrm{dB}$ and $16.08 \mathrm{~dB}$, respectively. The path-loss exponent $\gamma$ set is $\{3,4\}$. The shadowing deviation $\sigma$ is considered as $8[\mathrm{~dB}]$.

Figure 3 investigates the performance of the IoT-IVWSNs in terms of the average throughput. We notice that the average throughput of the network declines with the increase in distance and decrease in transmission power (Pt), as exhibited in Figure 3(a). This is understandable according to the path-loss model where the path loss increases with distance and the effect of the log-normal shadowing involves a fluctuation of the time of the received power, which can further degrade the performance of the communication $[44,45]$. Due to this fluctuation, the received power may go below the Receiver Sensitivity (Rs) (-95 dBm), thus the CU considers the received signal as noise that leads to a lower throughput. Again, we observe that the performance of the network is better in the case of the Rice fading compared to the Rayleigh fading because of the presence of a dominating signal component, as depicted in Figure 3(b).

Table 2. Communication Parameters

\begin{tabular}{|c|c|}
\hline Name & Value \\
\hline$P_{t}$ & $-15 \mathrm{dBM}, \mathrm{We}$ have mentioned in the previous section \\
\hline$C_{f}$ & $2.4 \mathrm{GHz}$ (ISM band), that is used for a sensor node [7] \\
\hline$R_{s}$ & $\begin{array}{c}\text { The reception threshold of the Coordinator of ZigBee is } \\
\text { set equal to }-95 \mathrm{dBm} \text {, it is usually for the ZigBee } \\
\text { Module [7] }\end{array}$ \\
\hline $\begin{array}{l}\text { Transmission Period } \\
\text { Packet size }\end{array}$ & $\begin{array}{l}120 \mathrm{~ms} \text { and } 60 \mathrm{~ms} \text { for low and high traffic, respectively } \\
210 \mathrm{bits}\end{array}$ \\
\hline Channel & $\begin{array}{l}\text { The path loss exponent for Rayleigh fading with NLOS } \\
\text { is } \gamma=4 \\
\text { The shadowing deviation is } 8 d B \text {. This value is } \\
\text { appropriate for intra-vehicle communication [7]. }\end{array}$ \\
\hline $\begin{array}{l}\text { Acknowledgement Wait } \\
\text { Duration }\end{array}$ & 0.05 second \\
\hline $\begin{array}{l}\text { Number of } \\
\text { retransmissions }\end{array}$ & 5 \\
\hline $\begin{array}{l}\text { Minimum Backoff } \\
\text { Exponent }\end{array}$ & 3 \\
\hline $\begin{array}{l}\text { Maximum Number of } \\
\text { Backoffs }\end{array}$ & 4 \\
\hline $\begin{array}{l}\text { Channel Sensing } \\
\text { Duration }\end{array}$ & 0.1 second \\
\hline
\end{tabular}

To clarify the issue above, it is shown in Figure 4(a) the effect of log-normal shadowing, with $\sigma=8 \mathrm{~dB}$, on the received power when the ED transmits with power $\mathrm{Pt}$ $=25 \mathrm{dBm}$ and $\gamma=3$. From the figure, it can be clearly noticed that as the distance increases, it increases the probability that the received power level falls below the RS, even when the average received power is greater than the threshold [46]. Moreover, a 
packet may be discarded, even if its received power level is greater than the threshold, but the Signal-to-Noise Ratio (SNR) is not sufficient for correcting the packet [47]. In fact, if the SNR is not sufficiently high, it can happen that a number of bits within the packet are incorrect. Unlike Figure 4(a), when $\gamma=4$, the effects of shadowing can bring the received power below the RS even when the distance between the CU and ED is 2 meters, as shown in Figure 4(b). Based on the above discussion, the considered communication parameters are summarised in Table 2.

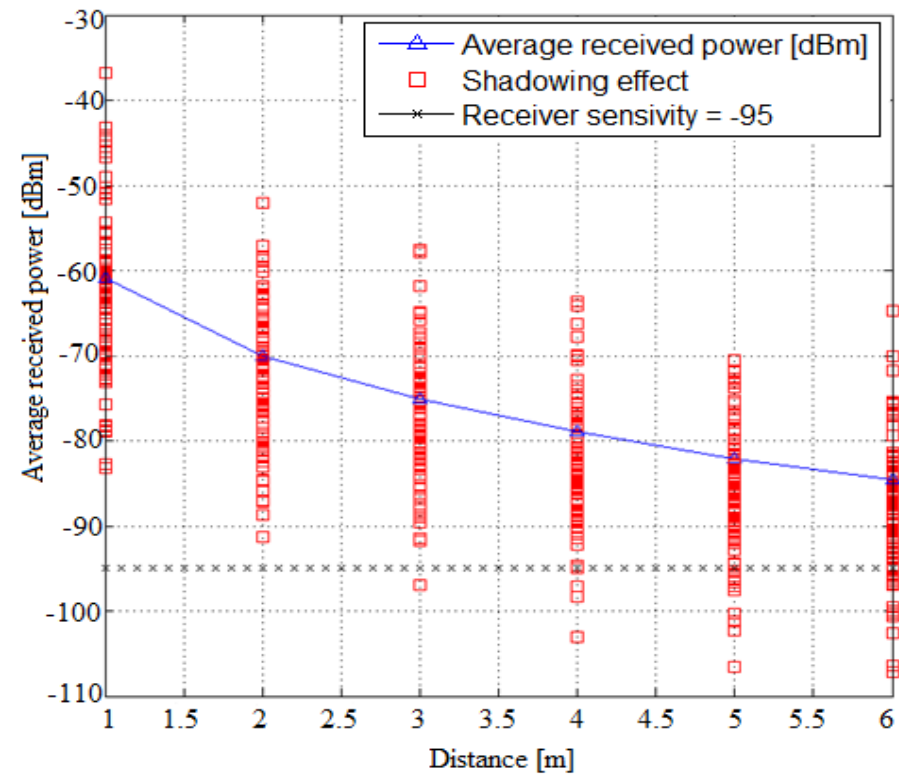

(a)

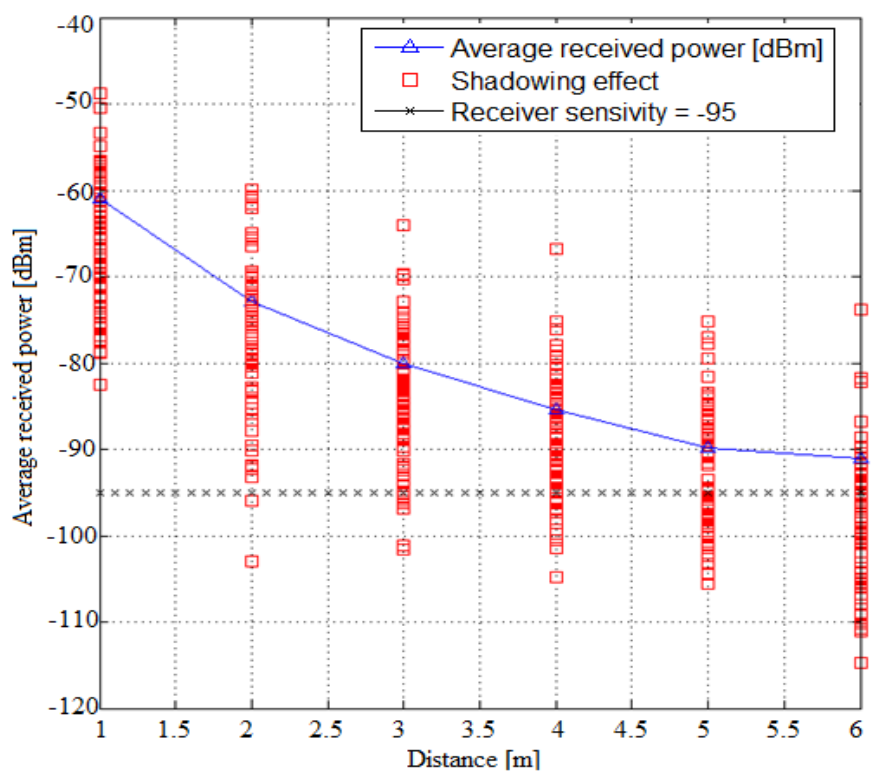

(b)

Figure 4. Effects of Log-normal Shadowing on the received power (a) Path-loss exponent $\gamma=3$; (b) Path-loss exponent $\gamma=4$.

\section{Performance Evaluation}

In this section, we investigate the performance of IoT-IVWSNs by varying the traffic load and network nodes, since these features play an important role after introducing the IoT 
concept on IVWSNs in order to address the scalability issue $[48,49]$. The suitability of the communication parameters is justified in the previous section, as listed in Table 2. In order to investigate the network performance, we have used the discrete event simulator, OPNET, with the ZigBee module. Four types of traffic load are considered in this experiment, namely Low-Traffic-Load (all are 120ms EDs), Medium-Traffic-Load (70\% $120 \mathrm{~ms}$ and 30\% 60ms EDs), Relatively-High-Traffic-Load (50\% 120ms and 50\% 60ms EDs) and High-Traffic-Load (30\% 120ms and 70\% 60ms EDs). Furthermore, to vary the network size, we consider four types of scenarios i.e., Scenario-I, Scenario-II, ScenarioIII and Scenario-IV which consist of 50, 70, 90 and 110 EDs, respectively.

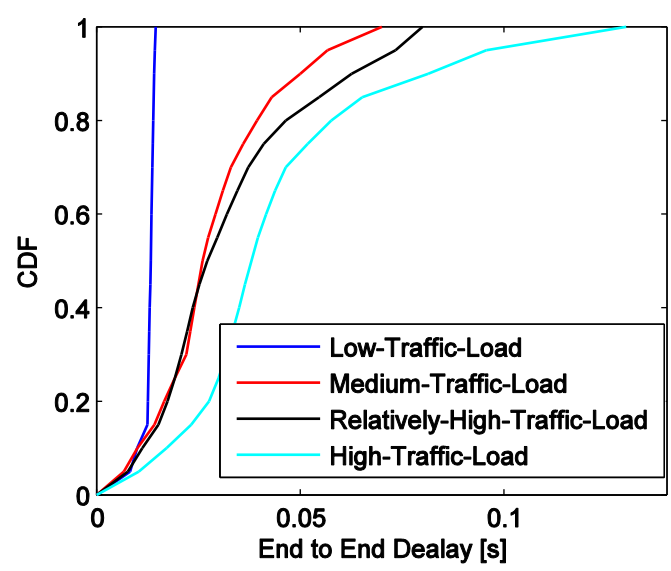

(a)

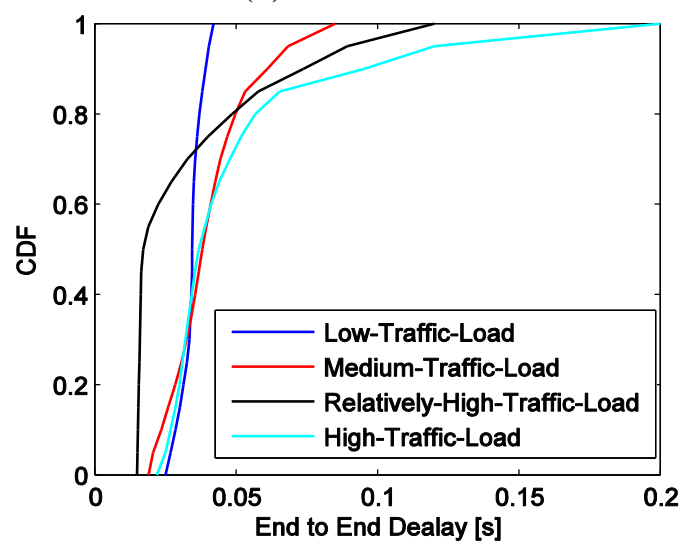

(c)

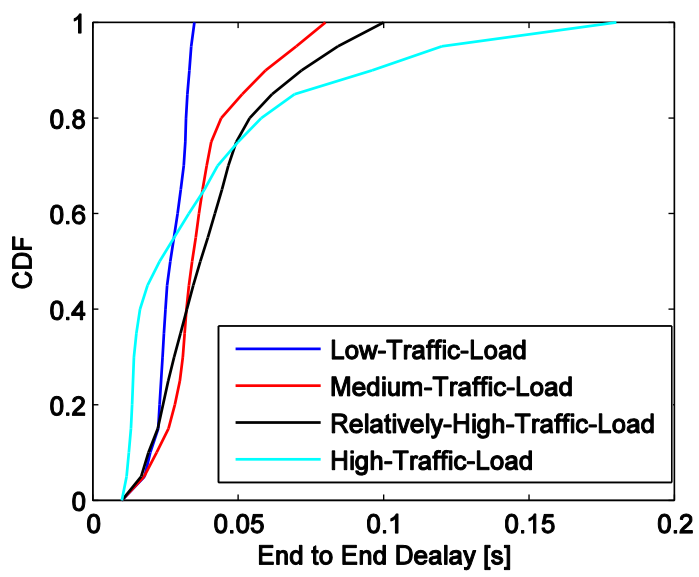

(b)

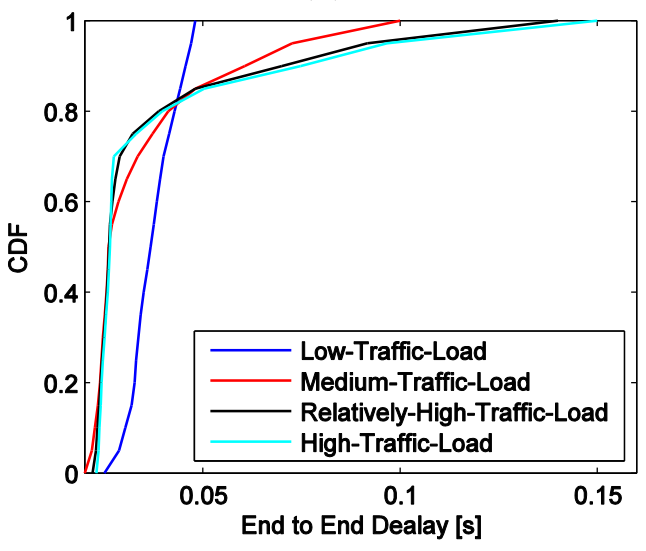

(d)

Figure 5. CDF versus End to End Delays: varying Traffic-Load and Network Size (a) Traffic-Load varies in Scenario-I; (b) Traffic-Load varies in Scenario-II; (c) TrafficLoad varies in Scenario-III; (d) Traffic-Load varies in Scenario-IV.

From Figure 5, we noticed that as the number of EDs and traffic load increased in IoT-IVWSNs, the CDF extends to the right implying rising delay times. Performance worsens when the number of EDs as well as the traffic load increases, which bring about collision among the packets [50]. When the collision occurs among the packets of ED, the ED needs to wait until the next allocated time slot begins and the channel is free. It again tries to retransmit the failed packet that already underwent collision previously. The new re-transmissions may again cause another collision. This recursive procedure is 
clarified in the CSMA-CA protocol. From the above discussion, it can be noted that the end-to-end delay of the network increases when the number of collisions rises among the packets [30]. In addition, with the increasing number of EDs with high traffic load, the congestion continues to grow for IoT-IVWSNs. In fact, this network can handle the traffic up to a certain point, when the traffic increases beyond that point, the network begins to enter into congestion. As a result, a number of packets of ED will be delayed to reach the destinations. Increased congestion in the network implies that more packets will be delayed to reach the destination. We also noticed distorted results. While with the increasing of traffic load, CDF becomes lower than the low traffic load scenario. This situation of congestion decreases the end-to-end delay that causes the different trajectories of the CDF curves, since in OPNET the end-to-end estimated delay depends on the of packets that already reached their destination.

\section{CONCLUSIONS}

In this paper, we investigated the performance of IoT enabled Intra-Vehicular Wireless Sensor Networks. The overview of IoT-IVWSN is presented considering its architecture, components, suitable existing technologies and the design challenges. The communication link between the $\mathrm{ED}$ and the $\mathrm{CU}$ is analysed to select the suitable communication parameters. The selected parameters were obtained as: transmit power $\mathrm{Pt}$ $=-15 \mathrm{dBm}$, channel frequency $\mathrm{Cf}=2.4 \mathrm{GHz}$, transmission period $=60-120$ milliseconds, packet size $=210$ bits, and channel path loss exponent $\gamma=4$. Using these parameters, we design the network scenario and investigate its performance by varying the traffic load with an average inter-arrival time of 60 to 120 milliseconds and network size with 50 to 110 nodes. Our test results demonstrate that the delay in packet transmission increases due to higher traffic loads and a larger number of end nodes. This result shows that the existing MAC protocol is not scalable i.e., it works well for a small network but it is not suitable for a large network. The behaviour of the scalability of IVWSNs significantly changes due to collision, congestion and collaboration in the network. A true cross-layer design could provide the best solution for such network, which would be the future direction of this research.

\section{ACKNOWLEDGEMENTS}

This work is partially supported by RDU (RDU1403167) project founded by University Malaysia Pahang and DRIVEr monitoring: technologies, methodologies, and IN-vehicle Innovative systems for a safe and eco-compatible driving (DRIVE IN2) founded by the Italian national program.

\section{REFERENCES}

[1] Gubbi J, Buyya R, Marusic S, Palaniswami M. Internet of Things (IoT): A vision, architectural elements, and future directions. Future Generation Computer Systems. 2013;29:1645-60.

[2] Borgia E. The Internet of Things vision: Key features, applications and open issues. Computer Communications. 2014;54:1-31.

[3] Da Xu L, He W, Li S. Internet of things in industries: A survey. IEEE Transactions on Industrial Informatics. 2014;10:2233-43. 
[4] Bello O, Zeadally S. Intelligent device-to-device communication in the internet of things. IEEE Systems Journal. 2016;10:1172-82.

[5] Chen K-C, Lien S-Y. Machine-to-machine communications: Technologies and challenges. Ad Hoc Networks. 2014;18:3-23.

[6] Tuohy S, Glavin M, Hughes C, Jones E, Trivedi M, Kilmartin L. Intra-vehicle networks: A review. IEEE Transactions on Intelligent Transportation Systems. 2015;16:534-45.

[7] Rahman MA. Design of wireless sensor network for intra-vehicular communications. International Conference on Wired/Wireless Internet Communications: Springer; 2014. p. 29-40.

[8] Azpilicueta L, Iturri PL, Aguirre E, Astrain JJ, Villadangos J, Zubiri C, et al. Characterization of wireless channel impact on wireless sensor network performance in public transportation buses. IEEE Transactions on Intelligent Transportation Systems. 2015;16:3280-93.

[9] Huang T-Y, Chang C-J, Lin C-W, Roy S, Ho T-Y. Intra-vehicle network routing algorithm for wiring weight and wireless transmit power minimization. The 20th IEEE Asia and South Pacific Design Automation Conference; 2015. p. 273-8.

[10] Jin Y, Kim KJ, Kwak KS. Performance analysis of UWB intra-vehicle transmitted-reference communication systems. 2011 IEEE Consumer Communications and Networking Conference; 2011. p. 277-81.

[11] Qu F, Li J, Yang L, Talty T. Measured channel capacity of SIMO-UWB for intravehicle communications. Proceedings of the 5th European Conference on Antennas and Propagation; 2011. p. 3056-60.

[12] Khuandaga G, Iqbal A, Kwak KS. Performance analysis of modulation schemes in Intra Vehicle Communications (IVC) channel. 13th International Conference on Advanced Communication Technology; 2011. p. 725-9.

[13] Bas CU, Ergen SC. Ultra-wideband channel model for intra-vehicular wireless sensor networks. 2012 IEEE Wireless Communications and Networking Conference; 2012. p. 42-7.

[14] Bas CU, Ergen SC. Ultra-wideband channel model for intra-vehicular wireless sensor networks beneath the chassis: from statistical model to simulations. IEEE Transactions on Vehicular Technology. 2013;62:14-25.

[15] Sadi Y, Ergen SC. Optimal power control, rate adaptation, and scheduling for UWB-based intravehicular wireless sensor networks. IEEE Transactions on Vehicular Technology. 2013;62:219-34.

[16] Song X, Li X, Tang W, Zhang W. A fusion strategy for reliable vehicle positioning utilizing RFID and in-vehicle sensors. Information Fusion. 2016;31:76-86.

[17] Ferdous RM, Reza AW, Siddiqui MF. Renewable energy harvesting for wireless sensors using passive RFID tag technology: A review. Renewable and Sustainable Energy Reviews. 2016;58:1114-28.

[18] Yue H, Zhang C, Pan M, Fang Y, Chen S. A time-efficient information collection protocol for large-scale RFID systems. Proceedings IEEE INFOCOM; 2012. p. 2158-66.

[19] Mandal K, Sen A, Chakraborty A, Roy S, Batabyal S, Bandyopadhyay S. Road traffic congestion monitoring and measurement using active RFID and GSM technology. 2011 14th International IEEE Conference on Intelligent Transportation Systems; 2011. p. 1375-9. 
[20] Kandhalu A, Xhafa A, Hosur S. Towards bounded-latency Bluetooth Low Energy for in-vehicle network cable replacement. 2013 International Conference on Connected Vehicles and Expo; 2013. p. 635-40.

[21] Bronzi W, Frank R, Castignani G, Engel T. Bluetooth low energy for intervehicular communications. 2014 IEEE Vehicular Networking Conference; 2014. p. $215-21$.

[22] Lin J-R, Talty T, Tonguz OK. On the potential of bluetooth low energy technology for vehicular applications. IEEE Communications Magazine. 2015;53:267-75.

[23] Qu F, Wang F-Y, Yang L. Intelligent transportation spaces: vehicles, traffic, communications, and beyond. IEEE Communications Magazine. 2010;48:13642.

[24] Iturri PL, Aguirre E, Azpilicueta L, Garate U, Falcone F. ZigBee radio channel analysis in a complex vehicular environment. IEEE Antennas and Propagation Magazine. 2014;56:232-45.

[25] Lu N, Cheng N, Zhang N, Shen X, Mark JW. Connected vehicles: Solutions and challenges. IEEE Internet of Things Journal. 2014;1:289-99.

[26] Lin J-R, Talty T, Tonguz OK. A Blind Zone Alert System Based on IntraVehicular Wireless Sensor Networks. IEEE Transactions on Industrial Informatics. 2015;11:476-84.

[27] Hwang S, Yu D. Remote monitoring and controlling system based on ZigBee networks. International Journal of Software Engineering and Its Applications. 2012;6:35-42.

[28] Chen S-K, Kao T, Chan C-T, Huang C-N, Chiang C-Y, Lai C-Y, et al. A reliable transmission protocol for zigbee-based wireless patient monitoring. IEEE Transactions on Information Technology in Biomedicine. 2012;16:6-16.

[29] Rahman MA. Reliability analysis of zigbee based intra-vehicle wireless sensor networks. International Workshop on Communication Technologies for Vehicles: Springer; 2014. p. 103-12.

[30] Rahman MA, Kabir MN, Azad S, Ali J. On mitigating hop-to-hop congestion problem in IoT enabled Intra-Vehicular communication. 4th International Conference on Software Engineering and Computer Systems; 2015. p. 213-7.

[31] Bhargav KK, Singhal R. Zigbee based VANETs for accident rescue missions in 3G WCDMA networks. IEEE South Asia Satellite Conference on Global Humanitarian Technology; 2013. p. 310-3.

[32] Parthasarathy D, Whiton R, Hagerskans J, Gustafsson T. An in-vehicle wireless sensor network for heavy vehicles. IEEE 21st International Conference on Emerging Technologies and Factory Automation; 2016. p. 1-8.

[33] Aalizadeh B, Asnafi A. Integrated bees algorithm and artificial neural network to propose an efficient controller for active front steering control of vehicles. International Journal of Automotive and Mechanical Engineering. 2016; 13(2):3476 - 3491

[34] Zamzamzadeh M, Saifizul A, Ramli R, Soong M. Dynamic simulation of brake pedal force effect on heavy vehicle braking distance under wet road conditions. International Journal of Automotive and Mechanical Engineering. 2016;13:355563.

[35] Iodice P, Senatore A. A numerical-experimental approach to assess emission performance of new generation engines during the cold transient. International Journal of Automotive and Mechanical Engineering. 2016;13:3599-612. 
[36] Nordin N, Adebambo H. Descriptive analysis of sustainable manufacturing indicators in Malaysian manufacturing firms. Journal of Mechanical Engineering and Sciences. 201610(2):2126-2133

[37] Fauzi F, Ghazalli Z, Siregar J. Effect of various kenaf fiber content on the mechanical properties of composites. Journal of Mechanical Engineering and Sciences. 2016;10:2226-33

[38] Ramasamy D, Yuan GC, Bakar RA, Zainal Z. Validation of road load characteristic of a sub-compact vehicle by engine operation. International Journal of Automotive and Mechanical Engineering. 2014;9:1820-31.

[39] Fatchurrohman N, Sulaiman S, Sapuan S, Ariffin M, Baharuddin B. Analysis of a metal matrix composites automotive component. International Journal of Automotive and Mechanical Engineering. 2015;11:2531-40.

[40] Mamdouhi H, Khatun S, Ahmadi K. An Intra wireless communication and remote control mechanism in Vehicular Ad-hoc network (VANET). IEEE Third UKSim European Symposium on Computer Modeling and Simulation, 2009; 2009. p. 505-10.

[41] Cheon J, Hwang H, Kim D, Jung Y. IEEE 802.15. 4 Zigbee-based time-of-arrival estimation for wireless sensor networks. Sensors. 2016;16:203.

[42] Peng C, Yue D, Fei M-R. A Higher energy-efficient sampling scheme for networked control systems over IEEE 802.15. 4 wireless networks. IEEE Transactions on Industrial Informatics. 2016;12:1766-74.

[43] Ahmed M, Saraydar CU, ElBatt T, Yin J, Talty T, Ames M. Intra-vehicular wireless networks. 2007 IEEE Workshops on Globecom; 2007. p. 1-9.

[44] Uddin MF. Throughput analysis of a CSMA based WLAN with successive interference cancellation under Rayleigh fading and shadowing. Wireless Networks. 2016;22:1285-98.

[45] Ross N, Schuhmacher D. Wireless network signals with moderately correlated shadowing still appear Poisson. IEEE Transactions on Information Theory. 2017;63:1177-98.

[46] Chen X, Ng DWK, Chen H-H. Secrecy wireless information and power transfer: Challenges and opportunities. IEEE Wireless Communications. 2016;23:54-61.

[47] Ganesh S, Amutha R. Efficient and secure routing protocol for wireless sensor networks through SNR based dynamic clustering mechanisms. Journal of Communications and Networks. 2013;15:422-9.

[48] Snigdh I, Gosain D. Analysis of scalability for routing protocols in wireless sensor networks. Optik-International Journal for Light and Electron Optics. 2016;127:2535-8.

[49] Abdmeziem MR, Tandjaoui D, Romdhani I. Architecting the internet of things: state of the art. Robots and Sensor Clouds: Springer; 2016. p. 55-75.

[50] Jabeen Q, Khan F, Khan S, Jan MA. Performance improvement in multihop wireless mobile adhoc networks. Journal Applied, Environmental, and Biological Sciences. 2016;6:82-92. 